University of Nebraska - Lincoln

DigitalCommons@University of Nebraska - Lincoln

May 2000

\title{
Quantitative measurement of guest-host interactions in supramolecular systems: A comparative Brillouin scattering study of the Dianin's compound clathrand and two of its isostructural clathrates
}

\author{
C.A. Sandstedt \\ University of Nebraska - Lincoln \\ D. Michalski \\ University of Nebraska - Lincoln \\ Craig J. Eckhardt \\ University of Nebraska - Lincoln, ceckhardt1@unl.edu
}

Follow this and additional works at: https://digitalcommons.unl.edu/chemistryeckhardt

Part of the Chemistry Commons

Sandstedt, C.A.; Michalski, D.; and Eckhardt, Craig J., "Quantitative measurement of guest-host interactions in supramolecular systems: A comparative Brillouin scattering study of the Dianin's compound clathrand and two of its isostructural clathrates" (2000). Craig J. Eckhardt Publications. 5. https://digitalcommons.unl.edu/chemistryeckhardt/5

This Article is brought to you for free and open access by the Published Research - Department of Chemistry at DigitalCommons@University of Nebraska - Lincoln. It has been accepted for inclusion in Craig J. Eckhardt Publications by an authorized administrator of DigitalCommons@University of Nebraska - Lincoln. 


\title{
Quantitative measurement of guest-host interactions in supramolecular systems: A comparative Brillouin scattering study of the Dianin's compound clathrand and two of its isostructural clathrates
}

\author{
C. A. Sandstedt, ${ }^{\text {a) }}$ D. Michalski, and C. J. Eckhardt ${ }^{\text {b) }}$ \\ Department of Chemistry, University of Nebraska-Lincoln, Lincoln, Nebraska 68588
}

(Received 30 August 1999; accepted 10 February 2000)

\begin{abstract}
Single, isomorphous crystals of the guest-free clathrand of Dianin's compound and two of its clathrates containing two ethanols and a heptanol, respectively, have been studied by Brillouin scattering to obtain their complete elastic constant tensors. The values found for individual elastic constants are related to microscopic interactions of the guests and host cage using the clathrand as the reference. Direct correlation of the interference of the guest heptanol molecule with the hexamer hydrogen-bonded network of the host cage to reduced stiffness of this clathrate is demonstrated. In contrast, the ethanol-containing clathrate is demonstrated to have negligible interaction of the guests on the host cage hydrogen bonding. Additionally, the ethanol guests are shown to provide a buttressing effect that increases the stiffness of the guest-host system in specific directions. The results are shown to be consistent with previous thermal conductivity and solid state NMR studies on these systems. The results demonstrate the use of macroscopic elastic constants in understanding the nature of the microscopic interactions in supramolecular crystals. (C) 2000 American Institute
\end{abstract} of Physics. [S0021-9606(00)70817-8]

\section{INTRODUCTION}

Beyond the chemistry focused on materials with strong covalent bonds lies supramolecular chemistry, which is centered upon complexes consisting of two or more chemical entities associated through van der Waals forces, hydrogen bonds, and/or intermolecular charge transfer interactions. ${ }^{1,2}$ These forces, although anisotropic, are neither as orientationally determined nor as strong as intermolecular forces. ${ }^{3}$ These interactions may provide structural as well as behavioral versatility allowing for an engineering of crystal properties. Significant progress in solid state chemistry is still needed to correlate the molecular structure and crystal packing modes with a solid's chemical and physical behavior. This knowledge also has wider ramifications due to its importance for the elucidation of the behavior of matter at the mesoscopic scale where a plethora of biologically active systems belong. ${ }^{4-6}$

Solid state reactions are another arena where recognition of the effects that play a crucial role, e.g., phonon-phonon and phonon-exciton couplings, involves understanding of mesoscale interactions and sensitivity to the importance of collective properties. ${ }^{7,8}$ Mechanochemistry, the study of reactions driven by mechanical energy, must also be understood in such a context. The phenomenon of detonation and, more generally, shock-induced chemical and physical processes, still lack formal scientific explanation. The time and length scales, as well as the nonergodic and nonequilibrium character of such processes, make a thermodynamical description quite formidable. The behavior of the phonon bath

\footnotetext{
${ }^{a}$ Current address: Department of Chemical Engineering, California Institute of Technology, Mail Code 210-41, Pasadena, California 91125.

${ }^{b)}$ Author to whom correspondence should be addressed.
}

of an explosive molecular system subjected to mechanical load is unknown. Proposed explanations range from exclusively stressing the role of electronic changes to models based on the flow of energy from acoustic to optic modes. ${ }^{9-11}$ Therefore, at this stage it is imperative not only to accumulate experimental data, but also to pose questions leading to new developments in solid state chemistry.

"Inclusion compound" refers to a wide range of systems in which one species (the guest) is spatially confined in nonrigorously defined cavities distributed within another species (the host). An upsurge of interest in inclusion compounds and their clathrate subgroup is due to recent emphasis on supramolecular systems that extends the paradigm of chemistry to include organization, composition, topology, and dynamics of molecular assemblies. ${ }^{12-18}$ Clathrates are crystalline inclusion compounds distinguished by their guest-host interaction and topological criteria. The guest molecules are confined by steric barriers created by the architecture of a crystalline host framework. The guest-host interaction is weak compared to conventional chemical bonding. ${ }^{19}$ Dynamic interactions have been usually neglected to simplify the physical picture of the encaged species to that of two noninteracting subsystems. ${ }^{19,20}$

The structure of inclusion compounds allows study and utilization of the effects of molecular confinement on the behavior of individual guest molecules, their small clusters, and even the entire guest-host assembly. ${ }^{21}$ Technological applications range from chemical analysis to pharmaceuticals. ${ }^{13,22}$ Clathrates serve as model systems in molecular recognition. ${ }^{23-25}$ The utilization of the particular geometry of the cavities can provide a novel environment for chemical reactions resulting in new products not obtainable from other phases. ${ }^{26}$ 
a)

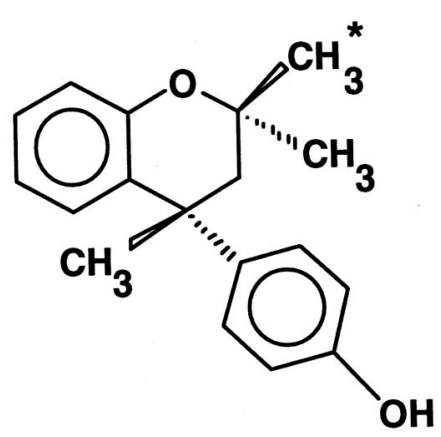

b)

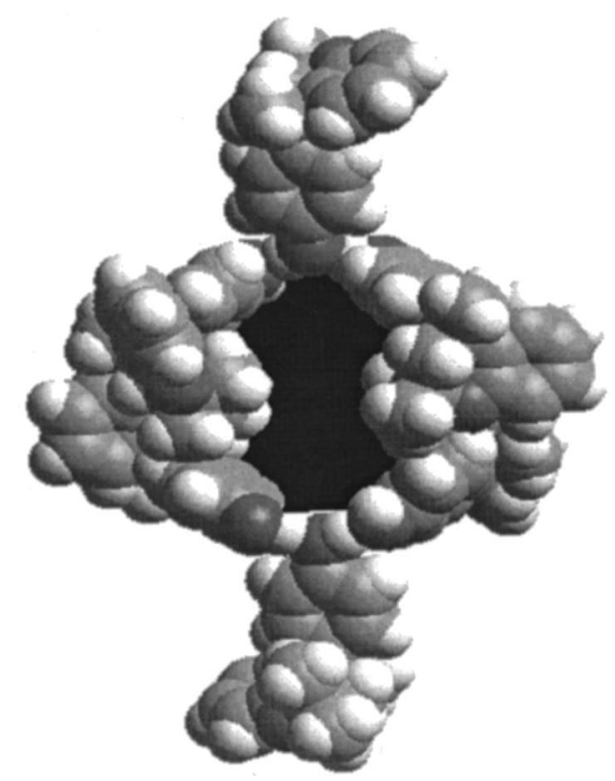

FIG. 1. (a) Molecular structure of Dianin's compound (4- $p$-hydroxylphenyl2,2,4-trimethyl chroman). (b) An ac plane projection of a single cage in Dianin's compound crystal. The Dianin's molecules in the front and back have been removed to show the effective hourglass contour of the cage.

For any system, however, it is the dynamics of the constituent species, directly related to thermodynamics, which determine most of its physical and chemical behavior. Recent experimental data and molecular dynamics calculations have shown the significant effect of these interactions on physical properties. ${ }^{27-29}$ It is of great importance to systematically study collective properties and relate them to the properties of the individual constituent species. This allows quantification of specific interactions and permits treating them as transferable observables. Thus a comprehensive study will identify macroscopic quantities by collective behavior of the chemical species.

Dianin's compound [4- $p$-hydroxyphenyl-2,2,4-trimethyl chroman, Fig. 1(a)] possesses an archetypal character that allows generalization of findings for the wider classes of inclusion systems, as well as exhibiting individual features favorable to isolation of a specific property. Dianin's compound possesses hydroxyl groups that form hydrogenbonded hexamers that alternately point up and down giving the supramolecular unit trigonal symmetry. Two supramolecular units, bound by van der Waals interactions, stack on top of each other creating an hourglass-shaped cavity where a guest molecule can reside [Fig. 1(b) $].{ }^{12,26}$ A unique feature of the Dianin's compound system of clathrates is the existence of an isostructural, unsolvated, host lattice, i.e., the Dianin's compound clathrand. This facilitates study of the effect of enclathration on collective properties.

Interesting physical behavior is reported for Dianin's inclusion compounds. In a solid state NMR study Haeberlin et al., investigated the motion of the two ethanol guests in the Dianin's clathrate cage. ${ }^{30}$ Previous x-ray crystallography measurements revealed that the two ethanol molecules segregate themselves between the two twin volumes of the cage. ${ }^{31}$ Although there is no apparent free volume for movement, the two guest molecules were observed to exchange positions. This requires that the host cage be flexible or perhaps an active participant in this exchange through some collective vibrational mode, which by its nature, would be a lattice mode.

Thermal conductivity measurements by White et al., on various crystalline Dianin's compound clathrates and the clathrand show glass-like thermal evolution of this property. ${ }^{32-34}$ A resonant scattering model was invoked that accounts for either host or guest mediated optic-acoustic coupling. There is also significant evidence suggesting that the type and number of guest molecules play important roles in determining the thermal conductivity of these systems.

The above considerations warrant investigation of the lattice dynamics of these inclusion compounds. The nature of the guest-host interaction is clearly crucial to such a study. This is tantamount to investigation of the lattice potential which, for solids, is revealed by their phonon spectra. Most relevant are the acoustic phonons whose macroscopic manifestation is found in the elastic constants. Elasticity directly reflects the intermolecular interactions and their anisotropy that determine the force constants between molecular groups and, therefore, the phonon dynamics. ${ }^{35-39}$

Crystallization decreases a system's symmetry and provides a new property, stiffness, which at the basic macroscopic level is reflected by Hooke's Law. Viewed from a microscopic perspective, a harmonic potential relates the intermolecular interactions between constituent species to their relative displacements within the unit cell. This is equivalent to the condition for existence of a condensed phase. Thus, the mechanical susceptibility intrinsically characterizes the solid phase. Neglecting elasticity and its associated derived quantities may lead to misconstruction of the origins and mechanisms of certain properties. For example, the topochemical postulate for solid state reactions does not consider the dynamical character of the crystalline environment. ${ }^{40,41}$

Herein we report the first concurrent experimental determination of the complete elastic constant tensors for three host-isomorphous clathrates based on Dianin's compound: the empty host clathrand, and the ethanol and heptanol clathrates. The clathrand provides the framework of reference. The choice of the other two systems was dictated by significant differences between the size, number, and behavior of the entrapped chemical species. Brillouin scattering provides a nondestructive probe of the elastic properties. The elasticity data elucidates the nature of the specific guest-host interactions in these systems. 
In the following section, essentials concerning the investigated Dianin's supramolecular systems and the experimental approach are described: synthesis, crystal growth, and lattice structure. The following sections discuss the physical basis of Brillouin scattering and its relation to elastic constant determination, instrumentation, measurements, and the determination of refractive indices. The remaining sections report the results of the measurements and discuss their implications with emphasis on the guest-host interactions and their energetics.

\section{SYNTHESIS, CRYSTAL PREPARATION, AND STRUCTURE}

\section{A. Synthesis}

Dianin's compound was prepared by the acid-catalyzed condensation of two molar equivalents of phenol with mesityl-oxide. ${ }^{42}$ Standard structural $\left({ }^{1} \mathrm{H}\right.$ and ${ }^{13} \mathrm{C}$ NMR, IR, and mass spectrometry), chromatographic (HPLC), and purification techniques (recrystalization and repeated sublimations) were performed to ensure that the synthesized product was sufficiently pure Dianin's compound.

\section{B. Crystal growth}

Clathrate crystals were grown from unsolvated Dianin's compound dissolved in the pure guest liquids at $40-45^{\circ} \mathrm{C}$. Solutions were sealed in a glass-jacketed container that permitted heating/cooling liquid to flow around the solution. The clathrand was prepared similarly from a decanol solution because previously reported measurements have shown that decanol is too large to fit inside Dianin's clathrate cavities. ${ }^{43,44}$ Solutions were heated to $45^{\circ} \mathrm{C}\left(60^{\circ} \mathrm{C}\right.$ for the clathrand) and cooled $\left(1{ }^{\circ} \mathrm{C} /\right.$ day $)$ to room temperature producing crystals of hexagonal, columnar morphology with average dimensions on the order of millimeters. The melting points determined using differential scanning calorimetry were $169.2 \pm 0.5^{\circ} \mathrm{C}$ for the ethanol and $163.5 \pm 0.5^{\circ} \mathrm{C}$ for the heptanol clathrates and $160.2 \pm 0.5^{\circ} \mathrm{C}$ for the clathrand.

All crystals were of exceptional optical quality: colorless, transparent, and exhibiting no cloudiness or internal cracks upon inspection under a microscope. They were single crystals, exhibiting no twinning, grain boundaries, or intergrowths by visual observation under a polarizing microscope. Faces were indexed using optical goniometry and matched the theoretical with an average accuracy of $\pm 0.5^{\circ}$.

\section{Crystal structure}

The three systems investigated crystallize in the trigonal $R \overline{3}$ space group with six individual Dianin's molecules per unit cell. Table I lists their crystallographic and general physical data. ${ }^{22,31,45}$ The only structural difference between the clathrate's of Dianin's complex is a guest-dependent variation in the unit cell dimensions.

The clathrates investigated have geometries based on the aforementioned hydrogen-bonded hexamer supramolecular unit in which the oxygen atoms form a distorted hexagon with alternate molecules of opposite handedness $(R$ and $S$ ) lying above and below the plane. ${ }^{12,22,46}$ Two such groups are
TABLE I. Crystallographic and general physical data of the three Dianin's compound based systems. The $R \overline{3}$ system is referenced to a hexagonal unit cell.

\begin{tabular}{llll}
\hline \hline \multicolumn{1}{c}{ Crystal data } & \multicolumn{1}{c}{$\begin{array}{c}\text { Clathrand } \\
\text { (Ref. 22) }\end{array}$} & \multicolumn{1}{c}{$\begin{array}{c}\text { Ethanol } \\
\text { (Ref. 31) }\end{array}$} & \multicolumn{1}{c}{$\begin{array}{l}\text { Heptanol } \\
\text { (Ref. 45) }\end{array}$} \\
\hline No. of guest molecules & 0 & 2 & 1 \\
Crystal system & Trigonal & Trigonal & Trigonal \\
Space group & $R \overline{3}$ & $R \overline{3}$ & $R \overline{3}$ \\
$a(\AA)$ & $26.94 \pm 0.010$ & $26.969 \pm 0.002$ & $27.12 \pm 0.03$ \\
$c(\AA)$ & $10.94 \pm 0.010$ & $10.990 \pm 0.002$ & $11.02 \pm 0.02$ \\
$\alpha(\mathrm{deg})$ & 90.00 & 90.00 & 90.00 \\
$\beta(\mathrm{deg})$ & 90.00 & 90.00 & 90.00 \\
$\gamma(\mathrm{deg})$ & 120.00 & 120.00 & 120.00 \\
$Z($ hexagonal $)$ & 18 & 24 & 21 \\
$\rho_{\text {calc }}\left(\mathrm{kg} / \mathrm{m}^{3}\right)$ & 1162.38 & 1223.40 & 1210.40 \\
Melting point $\left({ }^{\circ} \mathrm{C}\right)$ & $160.2 \pm .5$ & $169.2 \pm .5$ & $163.5 \pm .5$ \\
\hline \hline
\end{tabular}

stacked along the $c$-axis (hexagonal setting) so that the bulky, pendant side groups interlock to form the walls of the cage. The exceptional stability of the host structure is ascribed to hydrogen bonding and the shape of the host molecule. Figure 1(b) depicts a single cage of the Dianin's supramolecule. The equatorial waist is produced by inward pointing methyl groups of the individual Dianin's molecules [marked by an asterisk in Fig. 1(a)]. The threefold crystallographic axis (hexagonal setting) lies in the direction of the long-axis of the cage. The cage extends $\sim 11 \AA$ along the $c$-axis and is $\sim 6.2 \AA$ wide at its points of maximum extension at $z \sim 0.3$ and $z \sim 0.7$. The hydrogen-bonded hexamer possesses a diameter of $\sim 2.8 \AA$ and the inward pointing methyl groups reduce the waist diameter of the cage to $\sim 4.0$ Å.

The two ethanol molecules in the Dianin's compound host lattice are triply disordered as required by symmetry. In contrast, only one heptanol molecule is present in each cage and it assumes an extended gauche conformation along the cage axis in order to be included in the cage. The heptanol guests are triply disordered around the $c$-axis and vertically by pointing towards the top or bottom of the cage.

\section{BRILLOUIN SCATTERING: THEORY AND DETERMINATION OF ELASTIC CONSTANTS}

Brillouin scattering is the coherent Bragg reflection of monochromatic light from propagating fluctuations of the dielectric constant induced by the passage of thermally activated acoustic waves. ${ }^{47-49}$ The Brillouin shift equation for an anisotropic medium is

$$
\delta \omega_{e}= \pm \frac{v_{e} \omega_{i}}{c} \sqrt{n_{i}^{2}+n_{s}^{2}-2 n_{i} n_{s} \cos \theta},
$$

where $\delta \omega_{e}$ is the frequency shift of the inelastically scattered light, $v_{e}$ represents the velocity of the elastic wave, $\omega_{i}$ is the angular frequency of the incident light, $c$ is the speed of light, $\theta$ is the angle between the incident and scattered beams, and $n_{i}$ and $n_{s}$ represent the refractive index encountered by the incident and scattered light, respectively.

In the absence of body forces, the translational equation of motion for an acoustically vibrating medium referenced to a Cartesian coordinate system is 


$$
\rho \frac{\partial^{2} u_{i}}{\partial t^{2}}=c_{i j k l} \frac{\partial^{2} u_{l}}{\partial r_{j} \partial r_{k}}
$$

This equation of motion relates local Cartesian components of particle displacement from their equilibrium position to the spatial derivatives of $u$ and the elastic constants, $c_{i j k l} \cdot{ }^{47}$ Substitution of plane wave solutions into Eq. (2) and equating the group and phase velocities, as dictated by the relevant region of the Brillouin zone, results in the Christoffel determinant,

$$
\left[c_{i j k l} \hat{q}_{j} \hat{q}_{k}-\rho v_{e}^{2} \delta_{i l}\right]=0,
$$

where $\rho$ is the crystal's density, and $\hat{q}_{j}$ and $\hat{q}_{k}$ are the direction cosines of $\hat{q}_{e}$. Therefore, measurement of the velocity of acoustic waves propagating in unique crystal directions permits determination of a crystal's elastic constants. To circumvent the problem of solving nonlinear quadratic and cubic equations associated with measuring sound velocities along arbitrary directions, an iterative, least squares minimization appropriate to low symmetry crystals was employed. ${ }^{50,51}$ Since this approach does not rely on special propagation directions, experimental errors do not accumulate and ambiguities arising from solving coupled quadratic or cubic equations are eliminated.

\section{A. Brillouin scattering apparatus and measurements}

The scattering experiments were performed with a variable angle, four-circle light scattering apparatus described elsewhere. ${ }^{52}$ This instrument permits the incident light to rotate independently of the crystal and eliminates cutting the crystal to conform to a $90^{\circ}$ scattering configuration. Cutting induces bulk and surface defects which introduce significant errors in phonon direction determination and creates significant widening of the Rayleigh line that obscures the inelastically scattered Brillouin lines. The theoretical finesse is 78 with actual values between 50 and 68 routinely achieved. The issue of peak-order overlap commonly encountered in Fabry-Perot spectroscopy was addressed by the use of three different free spectral ranges (FSR) of $21.93 \mathrm{GHz}(0.731$ $\left.\mathrm{cm}^{-1}\right), 39.51 \mathrm{GHz}\left(1.317 \mathrm{~cm}^{-1}\right)$, and $45.18 \mathrm{GHz}(1.506$ $\mathrm{cm}^{-1}$ ). The FSR was standardized by employing both a polished cube of fused quartz and liquid benzene. ${ }^{53,54}$ Minimally, two crystals of each Dianin's clathrate were measured to establish reproducibility in the frequency shifts. All measured Brillouin spectra were the sum of at least 600 ten second Fabry-Perot interferometer scans. A typical Brillouin spectrum obtained from the heptanol clathrate is presented in Fig. 2.

Variable angle measurements require accurate knowledge of the refractive index and the refraction directions for a particular scattering geometry. Special care was taken to ensure that the incident and scattering polarizations were chosen along the crystal's ordinary refractive index or that for which the extraordinary beam's cross section was circular. ${ }^{55,56}$ This ensured applicability of Snell's Law and accurate determination of the scattering angle and phonon directions.

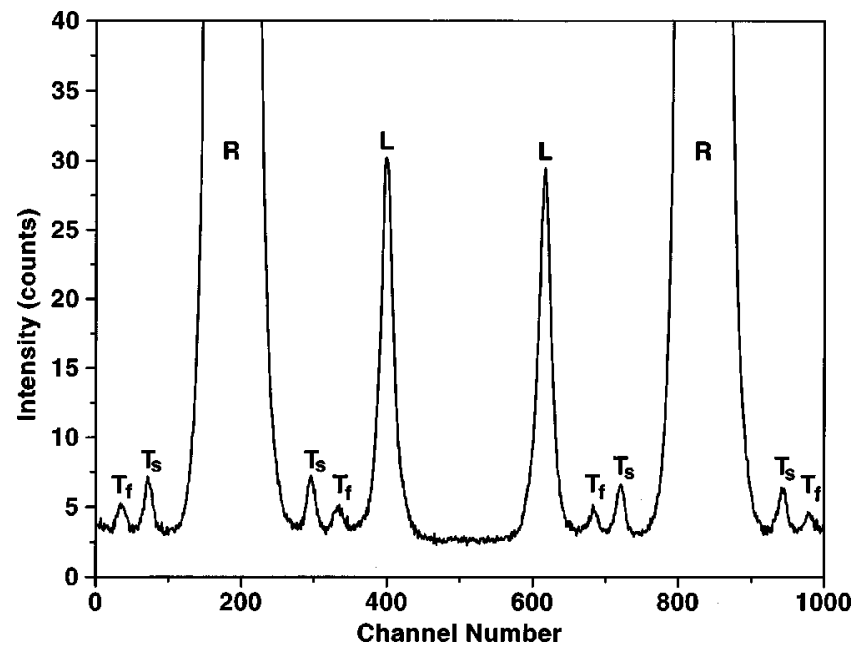

FIG. 2. $V V$ polarization Brillouin spectrum of the heptanol clathrate. The $\mathrm{FSR}=39.51 \mathrm{GHz}\left(1.317 \mathrm{~cm}^{-1}\right)$ and scattering is from the $(0.0762,0.9971$, $0)$ phonon. The peaks labeled $R, L, T_{f}$, and $T_{s}$ refer to the elastically scattered Rayleigh line and the inelastically scattered quasilongitudinal, fast transverse, and slow transverse acoustic modes, respectively.

\section{B. Determination of refractive indices}

Symmetry dictates uniaxial optical indicatrixes for the three Dianin's supramolecular complexes with the refractive indices isotropic in the $a b$ plane and the optic axis aligned parallel to both the crystallographic $c$-axis and to the six zonal side faces. Refractive indices were determined by polarized, specular reflection spectroscopy. ${ }^{57,58}$ Reflectivities were measured with the electric vector of the $514.5 \mathrm{~nm}$ incident light polarized parallel and perpendicular to the crystals' optic axes. At least three different areas on the same crystal face of each Dianin's crystal were measured followed by a $60^{\circ}$ rotation of the crystal about the crystallographic $c$-axis to permit reflectivity measurements on an adjacent crystal face. A minimum of two crystals of each of the three clathrates were so measured. Refractive indices for the clathrand and both the heptanol and ethanol Dianin's compound clathrates are presented in Table II.

\section{RESULTS AND DISCUSSION}

A total of 40, 54, and 51 nonequivalent Brillouin shifts for the clathrand and ethanol and heptanol clathrates, respectively, were measured. The stiffness constants for the three crystals are listed in Table III together with the average errors of the minimized sound velocities relative to the experimentally determined velocities. The matrices comply with the conditions for the mechanical stability of a crystal. ${ }^{35,38}$ To attach physical meaning to the elastic constants, a set of stress components is applied to the crystal in such a way that

TABLE II. Refractive indices for the three forms of Dianin's clathrate determined at $514.5 \mathrm{~nm}$ and $298 \mathrm{~K}$

\begin{tabular}{ccc}
\hline \hline Dianin's clathrate & $n_{z}$ & $n_{x y}$ \\
\hline Clathrand & $1.628 \pm 0.003$ & $1.602 \pm 0.001$ \\
Ethanol & $1.637 \pm 0.003$ & $1.610 \pm 0.006$ \\
Heptanol & $1.702 \pm 0.016$ & $1.663 \pm 0.020$ \\
\hline \hline
\end{tabular}


TABLE III. Elastic $\left(c_{I J}\right)$ and compliance $\left(s_{I J}\right)$ constants for the three Dianin's clathrates. The constants are expressed in Voigt notation and conform to an RII trigonal topology. Uncertainties in the elastic constants are 0.003 $\times 10^{10} \mathrm{~N} / \mathrm{m}^{2}$. The values for the corresponding compliance constants are included in parentheses. The average values (expressed in percents) of the differences between the observed and minimized values of the longitudinal and transverse acoustic mode velocities are presented in the last three rows of the table.

\begin{tabular}{llll}
\hline \hline $\begin{array}{c}\text { Elastic constants } \\
\left(\mathrm{N} / \mathrm{m}^{2}\right) \times 10^{10}\end{array}$ & \multicolumn{1}{c}{ Clathrand } & Ethanol clathrate & Heptanol clathrate \\
\hline$c_{11}\left(s_{11}\right)$ & $0.821(1.966)$ & $0.861(1.772)$ & $0.686(1.931)$ \\
$c_{12}\left(s_{12}\right)$ & $0.298(-0.322)$ & $0.351(-0.0486)$ & $0.239(-0.703)$ \\
$c_{13}\left(s_{13}\right)$ & $0.526(-0.830)$ & $0.429(0.651)$ & $0.210(-0.324)$ \\
$c_{14}\left(s_{14}\right)$ & $0.108(-0.752)$ & $0.103(-0.729)$ & $0.097(-0.867)$ \\
$c_{33}\left(s_{33}\right)$ & $1.061(1.765)$ & $0.846(1.840)$ & $0.797(1.425)$ \\
$c_{44}\left(s_{44}\right)$ & $0.333(3.641)$ & $0.318(3.123)$ & $0.294(4.008)$ \\
$c_{25}\left(s_{25}\right)$ & $0.060(-0.418)$ & $-0.009(0.068)$ & $0.024(-0.213)$ \\
$L$ & $0.5 \%$ & $0.2 \%$ & $0.3 \%$ \\
$T_{f}$ & $2.6 \%$ & $1.9 \%$ & $3.0 \%$ \\
$T_{s}$ & $1.2 \%$ & $2.2 \%$ & $2.8 \%$ \\
\hline \hline
\end{tabular}

all components of strain, except for one normal component or a pair of shear strain components, vanish. ${ }^{36}$ Such analysis for each of the experimentally determined elastic constants, followed by comparison between the three systems, and final correlation to the molecular structure allows a dynamical picture of these three crystals to be developed. As established by standard practice for RII trigonal crystal systems, the $c$ and $a$ crystallographic directions are coincident with the $z$ and $x$-axes of the elastic constant tensor, respectively, while the $y$-axis is rotated $30^{\circ}$ clockwise from the crystallographic b-direction. ${ }^{36,47}$

\section{A. Elastic constants}

The $c_{33}$ and $c_{44}$ elastic constants are determined from the velocity of the pure longitudinal and transverse phonons propagating along the $z(c)$-axis, respectively, and they are the only nonzero components of the RII trigonal elastic constant tensor that can be determined independently. The $c_{33}$ and $c_{44}$ elastic constants are not coupled to any other constants and thus directly probe the stiffness of the interactions along the $z(c)$-direction which is sensitive to the nature of the included guests.

The $c_{33}$ elastic constant couples the resulting uniaxial $z z$ (or $c c$ ) strain response to a normal compressional (or tensile) stress applied along the $z(c)$-axis and decreases in the order: clathrand, ethanol, and heptanol clathrates. This is consistent with the reported $\mathrm{x}$-ray lattice parameters which show that, compared to the clathrand, the cage is expanded in the $z$ (c)-direction for both the ethanol and heptanol clathrates, with heptanol at the extreme. Expansion of the lattice in the $z$ (c)-direction should produce a concomitant decrease in the van der Waals attraction between the two hexamer polycyclic ring systems that interlock to form a full cage. This leads to a softening of the lattice in the $z(c)$-direction, a trend reflected by the experimentally determined elastic constants.

The $c_{33}$ elastic constant for the trigonal system is uniquely determined by the equation $\rho v_{L, 001}^{2}=c_{33}$, where $v_{L, 001}$ is the velocity of the longitudinal elastic wave traveling along the [001] direction. Is the difference in the $c_{33}$ elastic constants between the three crystals due to a dramatic weakening of the supramolecule or merely governed by the change in density? As evident from Table I, the clathrand has the lowest density, but is stiffer along the $c$-axis by approximately $20 \%$ and $25 \%$ compared to the ethanol and heptanol clathrates, respectively. Furthermore the ethanol clathrate's density is only $\sim 1 \%$ larger than that of the heptanol clathrate; however, the ethanol clathrate's $c_{33}$ elastic constant is $6 \%$ greater. Thus, a change in density cannot account for differences in the stiffness along the $z(c)$-direction for these systems.

Cage hydrogen-bond interference is almost certainly applicable for the heptanol clathrate. X-ray crystallography shows that the heptanol guest molecule assumes a folded gauche confirmation in order to be included in the cage. ${ }^{46}$ The hydroxyl group of the heptanol molecule appearing at the top (or bottom) of the cage can directly compete with the hydrogen-bonds of the Dianin's supramolecular cage. The dramatic decrease in the $c_{33}$ elastic constant for the heptanol clathrate is consistent with such a cage disruption. Examination of the relative weakening of the $c_{33}$ elastic constant due to the two guests shows that the ethanol and heptanol clathrates' elastic constants show nearly the same relative weakening, $20 \%$ and $25 \%$, respectively. This suggests the competitive hydrogen bonding of the heptanol clathrate is somewhat more disruptive than for the ethanol.

The $c_{44}$ elastic constants for the three compounds mirror the decrease in stiffness of the lattice upon going from the empty clathrand to the clathrates. The $c_{44}$ elastic constant, which measures the resistance to shear deformation in the $y z$ plane by a $y$-polarized transverse wave propagating along the $z$ (c)-axis, is dependent only upon the density of the crystal and the speed of the transverse wave, $\rho v_{T, 001}^{2}=c_{44}$. For the same reasons as presented for $c_{33}$, the density cannot be the determining factor for the trend in shear stiffness for the three materials.

Although there is only a small difference in the relative weakening of $c_{33}$ by the ethanol and heptanol guests, this is not the case for $c_{44}$. While not as pronounced in magnitude as observed for $c_{33}$, the relative effects on $c_{44}$ are dramatically different with that of heptanol $(11.70 \%)$ being better than twice that observed for ethanol $(4.5 \%)$. This may be attributed to the passage of the heptanol through the waist of the cage.

The covalent diameter of the heptanol would be expected to be somewhat larger than that of the cage waist (4.1 $\AA$ ) and thus may be expected to structurally weaken the host lattice. ${ }^{59}$ In contrast, the ethanols residing in separate volumes of the hourglass cavities cause minimal dilation of the waist. Therefore, the ethanol clathrate's host lattice is much more similar to that of the clathrand. Thus, the decrease in the ethanol clathrate's $c_{44}$ might be solely due to expansion of the lattice in the $z(c)$-direction resulting in a reduction in the van der Waals attractive force. However, for the heptanol clathrate, an additional element in its softening is the threading of the guest heptanol molecules through the waist which perturbs the host-lattice of the precursor. For both the ethanol and heptanol clathrates the trend in softening for both the $c_{33}$ and $c_{44}$ elastic constants, or equivalently the speed of the 
longitudinal and transverse acoustic modes, is the same but with a slightly different underlying molecular origin.

Comparison of the $c_{11}$ and $c_{12}$ elastic constants shows an opposite trend compared to that previously discussed. For both constants, the ethanol is stiffer than the clathrand although, as before, the heptanol clathrate has the smallest values. The $c_{11}$ elastic constant may be regarded primarily as a measure of the stiffness in the plane normal to the threefold axis of the unit cell of the hydrogen-bonded hexamer plane. It relates the resulting uniaxial $x(a)$-strain response to a normal component of stress applied in the $x(a)$-direction. The value for the ethanol clathrate suggests the guest molecules are providing some stability to the lattice. This may be attributed to the presence of ethanols, more or less freely rotating, in each part of the cage. They may be expected to act as "fill material," thus effectively buttressing the mechanical strength of the cage. This is tantamount to arguing that the stiffening is due to density.

The role of guest molecules providing stability to the host lattice is a common feature of clathrate systems. For example, an empty, isomorphous, clathrand structure does not exist for the clathrate hydrates. Instead, in the absence of guest molecules, the hydrate collapses to its normal Ice Ih hexagonal structure. ${ }^{60}$ The hexa-host hexakis-(phenylthio) benzene (HPTB), like Dianin's compound, packs in the trigonal $R \overline{3}$ space group when it includes guest molecules such as $\mathrm{CCl}_{4}$ and $\mathrm{CBr}_{4}$, but forms a triclinic cell in the absence of a guest molecule. ${ }^{61}$

For cases when the $c_{15}$ elastic constant [resistance to $x z$ strain due to an $x(a)$-directed stress] is vanishingly small, such as with these systems, the $c_{11}$ elastic constant is uniquely determined by the velocity of the compressional, [100] longitudinal wave through the relation $\rho v_{L, 100}^{2}=c_{11}$. As with the $c_{33}$ and $c_{44}$ elastic constants, it is instructive to consider the role of a change in density upon these elastic constants. For $c_{11}$ the ethanol clathrate is stiffer than the clathrand by $4.6 \%$. However, the ethanol clathrate has a density $5 \%$ greater than that of the clathrand. Therefore, the apparent increase in stiffness for the ethanol clathrate is accounted for by the increase in density and, therefore, is just due to the "filling" of the available volume. However, the $c_{11}$ elastic constant for the heptanol clathrate is dramatically less than that for the clathrand and ethanol clathrate by $16.4 \%$ and $20.3 \%$, respectively. The heptanol clathrate's density is only $1 \%$ smaller than that for the ethanol clathrate causing a nondensity related weakening in the $x y(a b)$ plane, a result compatible with the previously reported increase in unit cell parameters for the heptanol clathrate. Because the heptanol cannot freely rotate and, thus, on average fill the cage, it cannot buttress the cage in the fashion that ethanol guests do. Further, the heptanol is also more disruptive of the hydrogen-bonded hexamer, which further decreases the stiffness in the $x y(a b)$ plane.

The $c_{12}$ elastic constant, which couples the resultant pure $y$-directed strain induced by a pure $x(a)$ tensile stress, follows the behavior of $c_{11}$ for each of the systems. Assuming again that $c_{15} \approx 0$, the Christoffel determinant reduces to $\rho v_{T, 010}^{2}$ $=\frac{1}{2}\left(c_{11}-c_{12}\right)$. The velocity appearing in this expression is that for an $x(a)$-polarized transverse wave and is the orthogo- nal particle displacement for a $y$-propagating compressional wave. Using the previous simplified expression for $c_{11}$, we have $c_{12}=\rho\left(v_{L, 100}^{2}-2 v_{T, 010}^{2}\right)$. Again densities should be examined. The ratio of the $c_{12}$ elastic constant of the clathrand to that of the ethanol clathrate is 0.85 , but that of the densities is 0.95 . Thus, the difference in elastic constants cannot be only due to a change in density, lending credence to the idea that the ethanol guest molecules reinforce the supramolecule against deformation in the $x y(a b)$ plane. The $c_{12}$ elastic constant for the heptanol clathrate substantiates the conclusion that the heptanol molecule interferes with the hydrogenbond network of the cages, creates steric expansion of the waist of the cage, and lends no support by failing to "fill" the cage.

The $c_{13}$ elastic constant couples a uniaxial strain in the $z$ (c)-direction to an applied normal-stress component in the $x$ (a)-direction. The elastic constants show a similar trend to that for $c_{33}$ and $c_{44}$ with the clathrand being the stiffest, the ethanol intermediate, and the heptanol the weakest. This further validates the argument that the presence of the longer, axially oriented heptanol molecule dramatically weakens the host lattice by expansion of the supramolecular cage and disruption of the hydrogen-bond network.

\section{B. Elastic anisotropy and the Cauchy ratios}

It is pertinent to assess the extent of the elastic anisotropy in these systems. Although phenomenological considerations stress the size and shape of potential guests, it is worth noting that the anisotropy of the interactions is related to crystal formation. The Cauchy ratios reveal angledependent forces which are related to the energetically favorable coupling between guests and hosts. ${ }^{37,39}$ The presence of these forces in other guest-host systems is related to the favorable growth of other clathrates from appropriate solutions.

The $R \overline{3}$ crystallographic system links the hexagonal and other trigonal crystal classes. The topology of the hexagonal elastic constant matrix is similar to that for $R \overline{3}$ with the only difference being that $c_{14}$ and $c_{15}=0 .{ }^{37,62}$ The elastic topology of the trigonal classes is divided into two types: space groups $R 32, R \overline{3} \mathrm{~m}$, and $R 3 \mathrm{~m}$ designated $\mathrm{RI}$; and space groups $R 3$ and $R \overline{3}$ comprising the RII topology. ${ }^{35}$ The only difference between these different trigonal elastic constant matrices is that for RI $c_{15}=0$. Therefore, the $c_{14}$ and $c_{15}$ constants are a measure of the degree of variance between the symmetries of elastic anisotropy and that of the crystal lattice.

The overall elastic anisotropy is assessed from the ratios that stem from conditions of the appropriate components of the $c_{i j}$ matrix for an isotropic material. For an elastically isotropic RII system $c_{11}=c_{33}, c_{12}=c_{13}, 2 c_{44}=\left(c_{11}-c_{12}\right)$ and $c_{14}=c_{15}=0$. Table IV lists the anisotropic factor ratios for the three Dianin's systems. These clearly show the anisotropic elastic nature of these three systems even when neglecting the measured nonzero contributions from $c_{14}$ and $c_{15}$. The ethanol adduct of Dianin's compound is the most isotropic with the smallest $c_{15}$. This is consistent with the picture of essentially freely moving ethanols acting to buttress the cages, but having a diminished effect on the 
TABLE IV. Anisotropic factor ratios for the three Dianin's clathrates.

\begin{tabular}{lccc}
\hline \hline & $\frac{c_{11}}{c_{33}}$ & $\frac{c_{12}}{c_{13}}$ & $\frac{2 c_{44}}{\left(c_{11}-c_{12}\right)}$ \\
\hline Clathrand & 0.772 & 0.568 & 1.274 \\
Ethanol & 1.017 & 0.819 & 1.247 \\
Heptanol & 0.861 & 1.135 & 1.134 \\
\hline \hline
\end{tabular}

hydrogen-bonded ring binding the cage hexamer. It also supports the proposed role of the heptanol, because its orientation and lack of mobility significantly contributes to the elastic anisotropy.

The symmetry of Dianin's clathrate systems reduces the six Cauchy ratios to two, i.e., $\left(c_{13} / c_{44}\right)$ and $2\left[c_{12} /\left(c_{11}\right.\right.$ $\left.\left.-c_{12}\right)\right] .{ }^{36}$ Their values are 1.58 and 1.14 for the clathrand, 1.15 and 1.38 for the ethanol clathrate, and 0.72 and 1.07 for the heptanol clathrate, respectively. Deviation from unity shows the presence of angular and torsional forces within the crystals. Cauchy ratios $c_{13} / c_{44}$ exceeding unity have been associated with rotational-translational coupling in $\mathrm{N}_{2}, \mathrm{CO}$, and $\mathrm{Ar}-\mathrm{O}_{2}$ mixtures. ${ }^{63,64}$ This is the case for the clathrand and the ethanol clathrate which shows that this coupling can be ascribed to the supramolecular hexamers. The low value for the heptanol indicates that the guest significantly affects the collective behavior of the host lattice.

\section{Crystal energetics}

Crystalline inclusion compounds do not conform easily to the conventional description of a crystalline molecular solid. They are neither mixed crystals nor molecular solutions but are well defined crystals, in terms of their host framework, of at least two chemical species. The guests may be disordered thereby complicating formal treatment. Previously mentioned models of these systems, strongly influenced by structural studies, are of a rigid host lattice and independent guest molecules. This assumption has also been made for their statistical mechanical treatment. ${ }^{20}$

The conclusions from this study show that it is necessary to combine the behavior and the dynamics of constituent molecular species into a complete and consistent picture. The differences in the unit cell dimensions between the three clathrates allow viewing the crystallographic data of clathrates as a specific distortion of the Dianin's compound clathrand. The deformation of the host framework is gauged by the strain energy as if the clathrand was under external stress brought about by the internal forces ascribed to the guests. The relevant strains are computed from an assumed homogenous deformation of the precursor, i.e., the clathrand.

There are only three nonzero Langrangian strains for this system. ${ }^{35}$ The strain for the ethanol and heptanol clathrates are $e_{11}=e_{22}=0.11 \%, e_{33}=0.46 \%$ and $e_{11}=e_{22}=0.67 \%, e_{33}$ $=0.73 \%$, respectively. These values fall into a range dictated by a harmonic elastic response. The corresponding strain energies for the ethanol and heptanol clathrates are $1.6 \times 10^{2} \mathrm{~kJ} / \mathrm{m}^{3}$ and $11.7 \times 10^{2} \mathrm{~kJ} / \mathrm{m}^{3}$, respectively, and reflect the work done per volume to yield a homogeneous deformation from the clathrand's host lattice to the clathrates' unit cell dimensions. The stresses causing this change are $\sigma_{11}=\sigma_{22}=3.6 \times 10^{7} \mathrm{~N} / \mathrm{m}^{2}, \sigma_{33}=5.4 \times 10^{7} \mathrm{~N} / \mathrm{m}^{2}$ for the ethanol clathrate and $\sigma_{11}=\sigma_{22}=11.3 \times 10^{7} \mathrm{~N} / \mathrm{m}^{2}, \sigma_{33}=11.3$ $\times 10^{7} \mathrm{~N} / \mathrm{m}^{2}$ for the heptanol clathrate. These stresses may be viewed as the distribution of forces between the guests and the host supramolecular units.

Heptanol molecules seem to distort the cavities more symmetrically than do the ethanol guests. Much lower values for the ethanol clathrate reflect unconstrained mobility of the guests in the cages. A possible explanation of this surprising result may be that the ethanols at any one time exert unbalanced interactions in their separate cages whilst the heptanol forces are more evenly distributed due its disorder being static. Further comparison between these clathrates is stymied by the qualitative difference in how the voids are occupied, i.e., only a single heptanol per cavity compared to the two ethanols in the twin volumes of the hourglass void.

The internal crystal elastic response undoubtedly causes the guests to offset the elastic energy increase of the lattice. This also shows that macroscopic quantities may be understood from the microscopic standpoint as a measure of the internal strains and stresses that couple to their external counterparts.

This simple view can be formalized by means of an elastic dipole model wherein the elastic medium of the host clathrand is perturbed by the presence of elastic dipoles (guest molecules) which describe a shift in the structure and related forces with resulting affect on the elastic field of the medium. ${ }^{65,66}$ The elastic dipole reflects the distribution of forces and can be envisaged as those negative stresses that maintain constant strain in the system. Guest molecules, modeled as elastic dipoles embedded into the elastic field of the host framework, effect the described change of the unit cell dimensions.

The theoretical treatment of melting of supramolecular systems is as complex as the problem of their aggregation. However, the melting point is indicative of the energetics holding the lattice together. In these systems, the melting points are, in increasing order: clathrand, heptanol, and ethanol. Clearly, this sequence does not follow the general elastic properties. There is no need for it to do so. The stiffness is a measure of the response of the lattice to an external stress. While this is related to the lattice potential, just as the force constant is related to the potential binding a molecule, it is not the same.

It might be expected that for largely van der Waals crystals such as these, the density might be somewhat more indicative of the lattice energy. At least it indicates why the clathrand should be the lowest melting. The much smaller density differences seen for the ethanol and heptanol systems would not lead to significant difference in dispersion forces. The lower melting of the heptanol clathrate is consistent with the disruptive effects that guest has, which, apparently, nearly cancel the stabilization expected from inclusion of guests.

The heptanol clathrate permits another estimation of the energetics of the guest-host interaction. The distortion of the heptanol molecule from its stable linear configuration estimates the energy the molecule is willing to expend to enter 
the cage. This would place a lower bound on the energy of the interaction of the heptanol with the host framework. The isomerization of the heptanol to the antiform and related twisting can be estimated to be around $18 \mathrm{~kJ} / \mathrm{mol} .{ }^{67}$ As shown above, the energies associated with the distortion of the heptanol-containing framework from that of the clathrand are of the same order of magnitude. This suggests that the formation of this system's guest-host complex is dependent on the specifics of the guest-host interaction and that distortion of the host framework is likely as supported by these and structural observations.

\section{CONCLUSIONS}

The guest-host interactions for three isomorphous, crystalline, inclusion compounds have been measured quantitatively by determination of all the elements of their elastic constant tensors. The constants support a model where the heptanol clathrate lattice is less stiff than the ethanol clathrate and the guest-free clathrand. By comparison of the elastic constants for these three systems, it is clear that the heptanol system's decreased stiffness is due to significant guesthost interactions that disrupt the stability of the host. Specifically, the heptanol competes with the hydrogenbonded structure that is key to the formation of the host cage. In addition, the heptanol molecule's size causes further weakening by forcing the host cage to expand at its waist. In contrast, the ethanol clathrate is quite similar to the stiffness of the clathrand. From the value of certain constants it is clear that the presence of the ethanol molecules in the host cage actually strengthens the cage by buttressing the cage and increasing its resistance to distortion. The data further show that the ethanols interact minimally with the hydrogenbonded system that largely holds the host lattice together.

Investigation of the anisotropy of the elasticity indicates that the heptanol system is more symmetric in its distribution of strains than is the ethanol clathrate. The Cauchy relations further support this picture but additionally show that there are significant angular and torsional forces with the guesthost crystals.

Examination of the energetics indicates that the distortion energy of the heptanol host lattice is on the order of the deformation energy of the host lattice. The trends in the melting points are also consistent with the observations of the guest-host interactions determined from the various elastic constants.

This study demonstrates that the elasticity of inclusion compound crystals can be directly related to microscopic guest-host interactions. This permits quantitative evaluation of the energetics of these interactions and suggests such an approach can be extended to other solid supramolecular systems. For isostructural systems, the applicability is apparent. For systems crystallizing in various space groups, analysis of the heptanol system is more related.

The anisotropy of the elasticity and the Cauchy ratios are related to the growth of the clathrates from appropriate solutions and thus with extensive gathering of these measures, may provide a complementary method of analysis of crystal growth. The strain and stress fields that merge the dynamical behavior of the guests with the host framework can be re- lated to the distribution of forces between these entities. Concerted motions of guest and/or host molecules, either local or extended, can manifest themselves through specific rearrangements of molecules such as found for the ethanol clathrate.

These observations suggest that the host framework can be viewed as an elastic medium in which elastic dipoles associated with the guests are embedded. The results obtained here demonstrate the influence of structural variance of the constituent molecules on the elastic properties of the collective properties of the supramolecular system. This knowledge is especially relevant for the design of new materials.

\section{ACKNOWLEDGMENT}

This research was supported by the University of Nebraska-Lincoln Center for Materials Research and Analysis.

${ }^{1}$ J. M. Lehn, Pure Appl. Chem. 50, 871 (1978).

${ }^{2}$ J. M. Lehn, Angew. Chem. Int. Ed. Engl. 27, 89 (1988).

${ }^{3}$ R. Destro and A. Gavezzotti, Studies in Physical and Theoretical Chemistry, edited by M. Pierrot (Elsevier, Amsterdam, 1990), Vol. 69, p. 161.

${ }^{4}$ H. Urabe, H. Hayashi, Y. Tominaga, Y. Nishimura, K. Kubota, and M. Tsuboi, J. Chem. Phys. 82, 531 (1985).

${ }^{5}$ T. Weidlich and S. M. Lindsay, J. Phys. Chem. 92, 6479 (1988).

${ }^{6}$ H. Urabe, Y. Sugawara, M. Tsukakoshi, and T. Kasuya, J. Chem. Phys. 95, 5519 (1991)

${ }^{7}$ N. M. Peachey and C. J. Eckhardt, J. Phys. Chem. 97, 10849 (1993).

${ }^{8}$ N. M. Peachey and C. J. Eckhardt, J. Phys. Chem. 98, 7106 (1993).

${ }^{9}$ D. D. Dlott and M. D. Fayer, J. Chem. Phys. 92, 3798 (1990).

${ }^{10}$ A. Tokmakoff, M. D. Fayer, and D. D. Dlott, J. Phys. Chem. 97, 1901 (1993).

${ }^{11}$ J. J. Gilman, Philos. Mag. B 71, 1057 (1995).

${ }^{12}$ D. D. MacNicol, J. J. McKendrick, and D. R. Wilson, Chem. Soc. Rev. 7, 61 (1978)

${ }^{13}$ J. E. D. Davies, W. Kemula, H. M. Powell, and N. O. Smith, J. Inclusion Phenom. 1, 3 (1983).

${ }^{14}$ E. Weber and H. P. Josel, J. Inclusion Phenom. 1, 79 (1983).

${ }^{15}$ H. M. Powell, Inclusion Compounds, edited by J. L. Atwood, J. E. D. Davies, and D. D. MacNicol (Academic, London 1984), Vol. 1, p. 1.

${ }^{16}$ E. Weber, Topics in Current Chemistry, edited by E. Weber (SpringerVerlag, Berlin, 1988), Vol. 140, p. 1.

${ }^{17}$ G. M. Whitesides, J. P. Mathias, and C. T. Seto, Science 254, 1312 (1991).

${ }^{18}$ R. Bishop, Chem. Soc. Rev. 25, 311 (1996).

${ }^{19}$ J. H. van der Waals, Trans. Faraday Soc. 52, 184 (1956).

${ }^{20}$ J. H. van der Waals, Advances in Chemical Physics, edited by I. Prigigone (Interscience Publishers, New York, 1959), Vol. II, p. 1.

${ }^{21}$ J. M. Thomas and K. D. M. Harris, Studies in Organic Chemistry, edited by G. R. Desiraju (Elsevier, Amsterdam, 1987), Vol. 32, p. 179.

${ }^{22}$ G. Tsoucaris, Studies in Organic Chemistry, edited by G. R. Desiraju (Elsevier, Amsterdam, 1987), Vol. 32, p. 209.

${ }^{23}$ R. Arad-Yellin, B. S. Green, M. Knossow, and G. Tsoucaris, Inclusion Compounds, edited by J. L. Atwood, J. E. D. Davies, and D. D. MacNicol (Academic, London, 1984), Vol. 3, p. 263.

${ }^{24}$ D. Worsh and F. Vogtle, Topics in Current Chemistry, edited by E. Weber (Springer-Verlag, Berlin, 1987), Vol. 140, p. 21.

${ }^{25}$ J. Szejtil, Inclusion Compounds, edited by J. L. Atwood, J. E. D. Davies, and D. D. MacNicol (Academic, London, 1984), Vol. 3, p. 331.

${ }^{26}$ J. E. D. Davies, Inclusion Compounds, edited by J. L. Atwood, J. E. D. Davies, and D. D. MacNicol (Academic, London, 1984), Vol. 3, p. 37.

${ }^{27}$ P. M. Rodger, Mol. Simul. 5, 315 (1990).

${ }^{28}$ P. M. Rodger, J. Phys. Chem. 94, 6080 (1990).

${ }^{29}$ R. M. Lynden-Bell, Mol. Phys. 79, 313 (1993).

${ }^{30}$ T. Bernhard, H. Zimmermann, and U. Haeberlein, Mol. Phys. 77, 1123 (1992).

${ }^{31}$ J. L. Flippen, J. Karle, and I. L. Karle, J. Am. Chem. Soc. 92, 3749 (1970).

${ }^{32}$ M. Zakrzewski and M. A. White, Phys. Rev. B 45, 2809 (1992).

${ }^{33}$ D. Michalski and M. A. White, J. Phys. Chem. 99, 3774 (1995). 
${ }^{34}$ W. Abriel, A. duBois, M. Zakrzewski, and M. A. White, Can. J. Chem. 68, 1352 (1990).

${ }^{35}$ J. F. Nye, Physical Properties of Crystals (University Press, London, 1957).

${ }^{36}$ A. I. Kitaigorodsky, Molecular Crystals and Molecules (Academic, New York, 1973).

${ }^{37}$ M. Born and H. Kun, Dynamical Theory of Crystal Lattices (Oxford University, London, 1954).

${ }^{38}$ D. C. Wallace, Thermodynamics of Crystals (Wiley, New York, 1972).

${ }^{39}$ G. Venkataraman, L. A. Feldkamp, and V. C. Sahni, Dynamics of Perfect Crystals (MIT Press, Cambridge, 1975).

${ }^{40}$ M. D. Cohen and G. M. J. Schmidt, J. Chem. Soc. 1964, 1996.

${ }^{41}$ M. D. Cohen, G. M. J. Schmidt, and F. Sonntag, J. Chem. Soc. 1964, 2000 .

${ }^{42}$ W. Baker, A. J. Floyd, J. F. W. McOmie, G. Pope, A. S. Weaving, and J. H. Wild, J. Chem. Soc. 1956, 2010.

${ }^{43}$ M. Zakrzewski and M. A. White, Phys. Rev. B 45, 2809 (1992).

${ }^{44}$ F. Lee, E. Gabe, J. S. Tse, and J. A. Ripmeester, J. Am. Chem. Soc. 110, 6014 (1988).

${ }^{45}$ J. L. Flippen and J. Karle, J. Phys. Chem. 75, 3566 (1971).

${ }^{46}$ J. L. Atwood, J. E. Davies, and D. D. MacNicol, Inclusion Compounds (Academic, London, 1984), Vol. 2.

${ }^{47}$ H. Z. Cummins and P. E. Schoen, Laser Handbook, edited by F. T. Arecchi and E. O. Schulz-Dubois (North-Holland, Amsterdam, 1972), p. 1029.

${ }^{48}$ I. L. Fabelinski, Molecular Scattering of Light (Plenum, New York, 1968).

${ }^{49}$ R. S. Krishnan, The Raman Effect, edited by A. Anderson (Marcel Dekker, New York, 1971), Vol. 1, p. 343.

${ }^{50}$ A. G. Every, Phys. Rev. B 22, 1745 (1980).
${ }^{51}$ K. H. Brose and C. J. Eckhardt, Chem. Phys. Lett. 125, 235 (1986).

${ }^{52}$ R. C. Dye, J. Sartwell, and C. J. Eckhardt, Rev. Sci. Instrum. 60, 2610 (1989).

${ }^{53}$ H. Z. Cummins and R. W. Gammon, J. Chem. Phys. 44, 2785 (1966).

${ }^{54}$ S. M. Shapiro, R. W. Gammon, and H. Z. Cummins, Appl. Phys. Lett. 9, 157 (1966).

${ }^{55}$ M. Born and E. Wolf, Principles of Optics: Electromagnetic Theory of Propagation, Interference and Diffraction of Light, 4th ed. (Pergamon, London, 1970).

${ }^{56}$ G. R. Fowles, Introduction to Modern Optics, 2nd ed. (Dover, New York, 1975).

${ }^{57}$ R. W. Dichtburn, Light (Blackie \& Son Limited, London, 1964), Vol. 2.

${ }^{58}$ C. J. Eckhardt and R. R. Pennelly, Chem. Phys. Lett. 9, 572 (1971).

${ }^{59}$ R. T. Morrison and R. N. Boyd, Organic Chemistry, 5th ed. (Allyn and Bacon, Boston, 1987)

${ }^{60}$ H. Kiefte, M. J. Clouter, and R. E. Gagnon, J. Phys. Chem. 89, 3103 (1985).

${ }^{61}$ D. M. Michalski, B. Mroz, H. Kiefte, M. A. White, and M. J. Clouter, J. Phys. Chem. 97, 12949 (1993).

${ }^{62}$ B. A. Auld, Acoustic Fields and Waves in Solids (Wiley, New York, 1973), Vol. 1.

${ }^{63}$ V. Askarpour, H. Kiefte, and H. Clouter, Can. J. Chem. 66, 541 (1988).

${ }^{64}$ S. F. Ahmad, H. Kiefte, M. J. Clouter, and M. D. Whitemore, Phys. Rev. B 26, 4239 (1982).

${ }^{65}$ R. T. Shuey and H. V. Beyeler, Z. Angew. Math. Phys. 19, 278 (1968).

${ }^{66}$ T. Luty and C. J. Eckhardt, J. Am. Chem. Soc. 117, 2441 (1995).

${ }^{67}$ E. L. Eliel, N. L. Allinger, S. J. Angyal, and G. A. Morrison, Conformational Analysis (American Chemical Society, Washington, D.C., 1965). 